C 2018 Universidad Nacional Autónoma de México, Facultad de Estudios Superiores Zaragoza.

Este es un artículo Open Access bajo la licencia CC BY-NC-ND (http://creativecommons.org/licenses/by-nc-nd/4.0/).

TIP Revista Especializada en Ciencias Químico-Biológicas, 21(Supl. 2): 30-42, 2018.

DOI: $10.22201 /$ fesz.23958723e.2018.0.153

\title{
Películas y recubrimientos comestibles funcionalizados
}

\author{
Luz Georgina Solano-Doblado, Liliana Alamilla-Beltrán \\ y Cristian Jiménez-Martínez* \\ Instituto Politécnico Nacional, Escuela Nacional de Ciencias Biológicas, \\ Departamento de Ingeniería Bioquímica, Unidad Profesional Adolfo López \\ Mateos, Av. Wilfrido Massieu Esq. Cda. Miguel Stampa s/n, Delegación \\ Gustavo A. Madero, Ciudad de México 07738, México. \\ E-mail: *crisjm_99@yahoo.com
}

\begin{abstract}
Resumen
Debido a las pérdidas en productos hortofrutícolas por plagas y manejo postcosecha inadecuado, en los últimos años se ha buscado el desarrollo y la producción de películas comestibles biodegradables como una forma de protección. Estas películas comestibles están hechas con materiales como carbohidratos, proteínas o lípidos, con características físicas y mecánicas similares a los envases no degradables, pero con la ventaja de funcionar como barreras bioactivas, ya que, mediante la adición de extractos vegetales, pueden presentar actividad antimicrobiana, protección contra la luz UV y visible, servir como acarreadores de antioxidantes y/o colorantes, mejorando con esto las características visuales del producto. Debido a estos atributos, han sido denominadas "películas inteligentes", ya que se ha observado un progreso considerable en su elaboración, como la presencia de sensores coloridos capaces de detectar cambios químicos y la presencia de microorganismos en los alimentos, mostrando si son o no, óptimos para el consumo. El objetivo de este trabajo es hacer una síntesis de diversas investigaciones, así como las tendencias recientes en el estudio y desarrollo de películas y recubrimientos comestibles, con énfasis en su aplicación a la cadena hortofrutícola y su efecto sobre productos frescos y mínimamente procesados.
\end{abstract}

Palabras Clave: películas, envases, protección, alimentos, desarrollo, innovación.

\section{Functionalized edible films and coatings}

\begin{abstract}
Pests and inadequate post-harvest handling has led to losses in horticultural products, therefore the development and production of edible biodegradable films has been sought as an alternative in recent years. These edible films are made with materials such as carbohydrates, proteins, or lipids, with physical and mechanical characteristics similar to the non-degradable packaging, but with the advantage of having a bioactive function as barriers; by adding vegetable extracts, edible biodegradable films may exert antimicrobial activity, protection against UV and visible light, against carriers of antioxidants and/or dyes, along with improving the visual characteristics of the product. Because of these attributes, edible biodegradable films have been referred to as "Intelligent films", since considerable progress has been observed in their elaboration, such as the presence of colorful sensors capable of detecting chemical changes and the presence of microorganisms in foods, revealing the state in which they are optimal and even if they are or not in an adequate state for consumption. The objective of this work is to make a summary of various research and current trends in the study and development of edible films and coatings, emphasizing their application in the horticultural chain and their effect on fresh and minimally processed foods.
\end{abstract}

Key Words: films, packaging, protection, food, development, innovation.

Nota: Artículo recibido el 8 de marzo del 2018 y aceptado el 20 de agosto del 2018. 


\section{INTRODUCCIÓN}

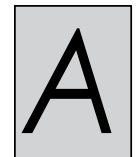
través de la historia y de los cambios necesarios de una moderna sociedad, los envases han jugado diferentes e importantes papeles, cubriendo nuevos requisitos y características. Dentro del desarrollo de sus funciones, los envases pueden clasificarse como: Primarios, que son los que tienen contacto directo con el alimento o productos; Secundarios, que contiene uno o varios envases primarios; Terciarios, que sirven para distribuir, unificar y proteger al producto a lo largo de la cadena comercial. Dentro de los primeros se encuentran los envases flexibles, que son elaborados de películas plásticas, papel, hojas de aluminio, laminaciones u otros materiales flexibles como co-extrucciones. Este tipo de envases no resiste la estiba, sin embargo, resulta práctico para el fácil manejo de otros productos. Dentro de éstos se encuentran las películas y recubrimientos comestibles, utilizadas para proteger productos hortofrutícolas antes y durante la postcosecha. Según la FAO, en el 2017 el desperdicio de alimentos perecederos fue de aproximadamente 1,300 millones de toneladas (FAO, 2018), estas mermas son provocadas por el inadecuado manejo postcosecha, ocasionado por daños físicos, microbiológicos y de almacenamiento (Maki-Díazet al., 2015). Los recubrimientos y películas comestibles, son una alternativa para mejorar y prolongar la calidad de los alimentos durante el manejo pre y postcosecha, así como el procesado, transporte y almacenamiento de los mismos (Espino-Díaz et al., 2010).

\section{Películas y recubrimientos comestibles}

La diferencia entre una película y un recubrimiento comestible es la forma de elaborarlos y aplicarlos en el alimento. Una película es una capa delgada de material comestible, formada por separado y que es colocada sobre una superficie nivelada para su posterior uso (Sharma \& Rao, 2015). Un recubrimiento se aplica sobre la superficie de un alimento, ya sea por inmersión en una disolución o por aspersión (Arredondo- Ochoa, 2012). Las películas están formadas por tres componentes: el polímero, el disolvente y finalmente el plastificante. Las técnicas comúnmente utilizadas para la elaboración de las películas y recubrimientos comestibles son las siguientes:

1. Por Eliminación del Disolvente.- En este proceso se forma y se estabiliza una estructura molecular por interacciones físicas y químicas. La disolución formadora del hidrocoloide comestible se incorpora en un disolvente (agua, etanol, ácido acético) que contiene a los aditivos como, plastificantes, agentes de reticulación o solutos; paso seguido se elimina el disolvente, con lo que se forma una capa delgada que se seca y que finalmente se puede desprender (Cagri, Ustunol \& Ryser, 2004).

2. Por Gelación Térmica.- La aplicación de un tratamiento térmico en este proceso permite la formación de un gel estable de estructura rígida. Ésta es utilizada generalmente para películas y recubrimientos elaborados a base de proteínas, descrita como un proceso en el que la desnaturalización térmica desestabiliza las moléculas de proteínas, las cuales dan lugar a la formación de un entramado estable entre partículas (Carmona Gallego, Cordobés, Guerrero Conejo, Martínez \& Partal, 2007).

3. Por Solidificación.-En este método las macromoléculas junto con el plastificante, son disueltas hasta su homogeneización y son vertidas en capas finas sobre moldes (Ávila-Sosa et al., 2012).

4. Por el método de "Casting".- Una vez realizada la disolución de los componentes de la película, se realiza la evaporación del disolvente a temperatura y humedad controladas, provocando la formación de la película (Escobar, Sala, Silvera, Harispe \& Márquez, 2009).

5. Por"electrospraying"(Pulverización electrohidrodinámica).Es un método de atomización del líquido formador de la película por medio de fuerzas eléctricas, en donde el líquido sale por una boquilla con un alto potencial eléctrico. La ventaja de este método es que las gotas que se obtienen son tan pequeñas que el tamaño de su partícula llega a medir incluso nanómetros, y la carga y el tamaño pueden controlarse en cierta medida por medios eléctricos (Jaworek \& Sobczyk, 2008).

5. Por Microfluidización.- Proceso en que las dispersiones se hacen pasar a través de microcanales, lo que permite la obtención de nanopartículas que otorgan a la película mejores características físicas, y posteriormente se aplica el método de casting para la formación de la película(Monroy-Villagrana, Cano-Sarmiento, Alamilla-Beltrán, Hernández-Sánchez \& Gutiérrez-López, 2014).

\section{BIODEGRADABILIDAD DE PELÍCULAS Y RECUBRIMIENTOS}

Sobre los productos poliméricos con los que se elaboran los recubrimientos y las películas comestibles, existen los poliméricos no degradables o sintéticos, que son elaborados a partir de compuestos petroquímicos, cuyos desperdicios permanecen en el ambiente por muchos años, produciendo un alto grado de contaminación y daños potenciales a la vida silvestre. Los biodegradables que son de origen natural, utilizados para la elaboración de películas y recubrimientos comestibles, permiten la obtención de materiales susceptibles a la degradación por la exposición a distintos factores del medio ambiente, que pueden ser tanto de naturaleza aeróbica como anaeróbica (Escobar, Sala, Silvera, Harispe \& Márquez, 2009).

La biodegradabilidad se define como la capacidad de un material de descomponerse en dióxido de carbono, metano, agua y componentes orgánicos o biomasa, cuyo mecanismo predominante es la acción enzimática de microorganismos que se miden por ensayos estandarizados, probados en períodos y condiciones de almacenamiento específicos (López, Rivas, Loaiza \& Sabino, 2010).

Tanto las películas como los recubrimientos comestibles pueden elaborarse de sustancias poliméricas naturales como 
el almidón (de fuentes como maíz, papa, yuca), mucílago de nopal y quitosano (López, Rivas, Loaiza \& Sabino, 2010), así como algunos hidrocoloides como las gomas arábiga y xantana, que siguen el ciclo de los polímeros biodegradables debido a la fuente de donde son extraídos, y sirven como base para producir un polímero con el cual se pueden fabricar películas, bolsas y empaques bioplásticos, para recolectar desperdicios orgánicos. Éstos se almacenan junto con el empaque, que se transforma en abono orgánico útil y como fertilizante para nuevas plantas (Ruiz-Avilés, 2006). Los retos en la investigación van dirigidos al uso de recubrimientos con este tipo de polímeros, para su aplicación industrial en la fabricación de bolsas, empaques o películas, y encontrar el menor tiempo de biodegradabilidad de cada elemento utilizado para este fin (Kean \&Thanou, 2010), además de una alternativa ecológica para sustituir los empaques plásticos comúnmente utilizados, que son causantes de la contaminación y tardan más de cien años en degradarse (Falguera, Quintero, Jiménez, Muñoz \& Ibarz 2011), contrario a las realizadas con polímeros naturales como el quitosano, las cuales han demostrado un tiempo mínimo de degradación de ocho días (López, Rivas, Loaiza \&Sabino, 2010).

\section{Usos y funciones de las películas y recubrimientos comestibles} El uso de películas y recubrimientos comestibles en alimentos, evitan la pérdida o ganancia de humedad que provoca una modificación en su textura y turgencia, retardan los cambios químicos como el color, aroma y valor nutricional, ya que actúan como barrera contra el intercambio de gases que influye en la estabilidad química y microbiológica, además de evitar el daño mecánico por manipulación (McHugh, 2000).

La aplicación de películas y cubiertas comestibles elaboradas con biomoléculas, también puede funcionar como un microsistema que ayuda a modificar las atmósferas del interior de los productos vegetales, lo que representa una alternativa para la conservación de productos hortofrutícolas frescos, al reducir significativamente la pérdida de peso, agua y el intercambio de gases, así como retrasar el envejecimiento y mejorar la calidad sensorial de éstos (Salinas Salazar, Trejo Márquez \& Lira Vargas, 2015).

\section{Propiedades físicas Y meCánicas de películas Y RECUBRIMIENTOS COMESTIBLES}

Las características físicas y mecánicas de las películas y recubrimientos dependen directamente de su formulación y de éstas, el uso final, dentro de las más importantes se encuentran:

\section{Barrera a gases}

Éstas dependen de las condiciones de humedad relativa que rodee a la película o recubrimiento comestible, y se busca controlar el intercambio de $\mathrm{O}_{2}, \mathrm{CO}_{2}$ y vapor de agua. En general la mayoría de los polímeros de fuentes naturales, poseen buenas propiedades de barrera, ya que son semi-permeables a los gases (Silva-Weiss, Ihl, Sobral, Gómez-Guillén \& Bifani, 2013).

\section{Permeabilidad al vapor de agua}

La permeabilidad de una barrera se calcula a partir de una combinación de la ley de Fick para la difusión y la de Henry para la solubilidad. Ésta es una medida de la facilidad con la que el vapor de agua puede penetrar en un material (Aider, 2010). La transferencia de vapor de agua, depende generalmente de la porción hidrofóbica de los componentes de la película o recubrimiento comestible, ya que a través del movimiento del vapor de agua en los polímeros, se controla la transferencia de humedad desde el producto al medio ambiente, por lo que se busca que sea lo más lento posible. Se han realizado estudios (Bósquez-Molina \& Vernon-Carter, 2005; Miranda, Cárdenas, López \& Lara-Sagahon, 2003) sobre la relación que existe entre la permeabilidad al vapor de agua y el tipo de componentes de la película o recubrimiento. Se ha observado relación entre el grosor de películas elaboradas con base de quitosano, y plastificantes como los polioles, glicerol, sorbitol, polietilenglicol encontrando que un incremento en el grosor aumenta la permeabilidad al agua (Trejo, Aragón \& Miranda, 2001; Arvanitoyannis, Kolokuris, Nakayama \& Aiba, 1997; Ramos-García et al., 2010).

\section{Barrera contra la luz, RUV, pérdida de color y opacidad}

La luz actúa como catalizador de procesos de oxidación y degradación en los compuestos nutricionales de los alimentos, por lo que para evitarlos, se adicionan componentes específicos que absorben la luz en el espectro de UV/Vis (Falguera, Quintero, Jiménez, Muñoz \& Ibarz, 2011). Las propiedades de barrera contra los efectos de la luz están relacionados con el color y la opacidad. En el caso de frutas y hortalizas, el brillo es una característica deseable, por lo que se busca obtener equilibrio entre la calidad sensorial y la nutrimental de estos alimentos (Márquez, Trillos, Cartagena \& Cotes, 2009). Las vitaminas liposolubles como la $\mathrm{E}$, incrementan la transparencia de las películas y recubrimientos, sin embargo, durante la vida de anaquel, permiten el paso de la luz, lo que cataliza la rancidez de los lípidos (Yang \& Paulson, 2000a). Para evitar ésto, se han utilizado extractos de plantas que dan color y opacidad a las películas, actúan como barrera para la luz y la degradación de ácido ascórbico, por lo tanto el oscurecimiento (Hong, Park \& Kim, 2000). Algunos ejemplos de estos extractos son los obtenidos del orégano y romero (Vartiainen, Vaha-Nissi \& Harlin, 2014).

\section{RESISTENCIA A LA TENSIÓN, ELONGACIÓN Y AL ROMPIMIENTO}

Estas propiedades están relacionadas con la estructura química del recubrimiento o película. La resistencia a la tensión (TS, MPa) se refiere a la máxima resistencia que presenta la película al estiramiento (Figura 1), mientras que la elongación al rompimiento $(\mathrm{EB}, \%)$ es la medida de la capacidad de estiramiento o flexibilidad que tiene la película, en ambas, se miden hasta que la película se rompe (Yang \& Paulson, 2000b). Dependiendo de las características que se busquen en la película o recubrimiento, pueden agregarse diferentes aditivos 


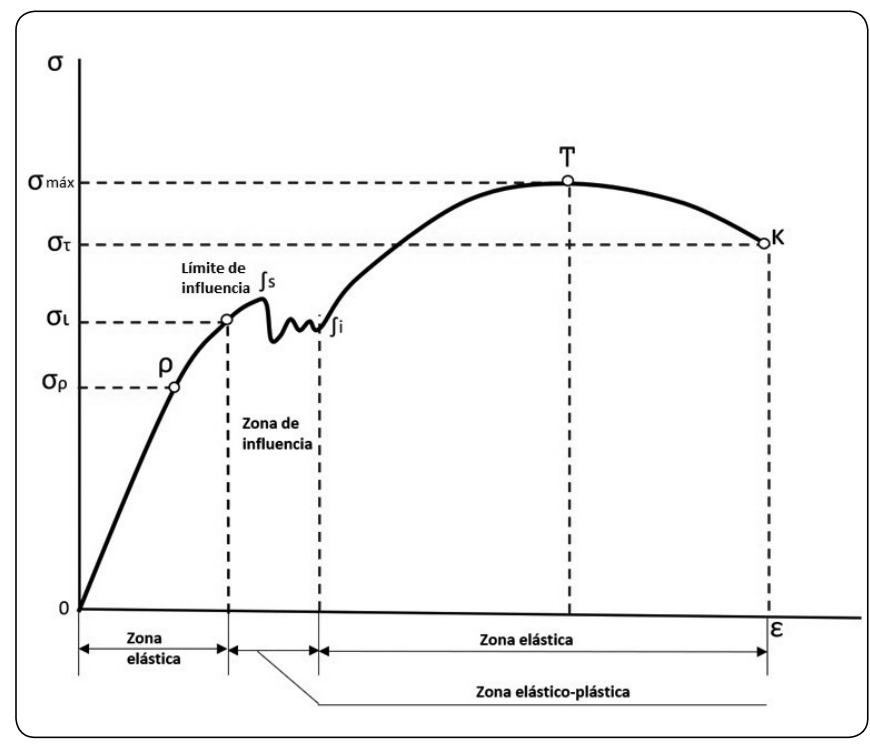

Figura 1. Zonas de elásticas y plásticas, resultado del esfuerzo del estiramiento en una película. Fuente: Hibberler (2006).

para mejorar estas propiedades, por ejemplo una película elaborada con gelatina y adicionada con $\alpha$-tocoferol reduce la elongación, debido a que la interacción de ambos compuestos disminuye el movimiento de las macromoléculas en la película (Jongjareonrak, Benjakul, Visessanguan \& Tanaka, 2008).

\section{INGREDIENTES FUNCIONALES UTILIZADOS EN PELÍCULAS Y RECUBRIMIENTOS COMESTIBLES}

Algunos Ingredientes funcionales utilizados en el diseño de películas y recubrimientos comestibles son: fármacos, vitaminas, antimicrobianos, saborizantes, colorantes y conservadores, éstos son importantes para mejorar la funcionalidad de las películas comestibles, alimentos procesados, así como para cosméticos y productos farmacéuticos (Sharma \& Rao, 2015). Estos ingredientes funcionales difieren en cuanto a su forma molecular, física, su polaridad y su masa molar. El uso de la nanotecnología ha permitido la incorporación de estos compuestos en las películas sin perder su funcionalidad, por lo cual se busca que la película:

1) Sirva como vehículo para acarrear el ingrediente funcional;

2) Proteja al ingrediente funcional de degradación química o biológica, durante el proceso de almacenamiento y utilización, ya que, durante estos estados, el ingrediente debe permanecer activo;

3) Sea capaz de controlar la liberación del ingrediente funcional a un ritmo específico, en las condiciones normales del alimento, es decir al $\mathrm{pH}$, a la temperatura natural y de almacenamiento;

4) Los ingredientes funcionales deben ser compatibles con la película y con otros componentes del sistema, al igual que con la fisicoquímica y cualitativamente con los atributos del alimento, es decir, que no altere negativamente la apariencia, textura, sabor y vida de anaquel.

Todos estos factores influyen en la eficacia y funcionalidad de la película (Aguilar, 2005), y para la liberación controlada y exacta de estos ingredientes se han desarrollado tecnologías como la encapsulación simple, emulsiones, coloides y matriz de biopolímeros (Weiss, Takhistov \& McClements, 2006).

\section{POLISACÁRIDOS UTILIZADOS EN LA FORMACIÓN DE LA MATRIZ ESTRUCTURAL DE PELÍ́CULAS}

Para la formación de la matriz estructural de una película o recubrimiento se utilizan componentes de naturaleza polimérica y de alta masa molar, y sobre ésta, pueden agregarse otros componentes que mejoran sus características o atributos. Los recubrimientos y películas comestibles son clasificados de acuerdo al material estructural que lo conforma y en caso de que el uso final sea la protección de alimentos, debe contener sustancias de alta calidad alimentaria, inocuas y de bajo costo. Dentro de ellos hay diversidad de compuestos que pueden ser utilizados, algunos ejemplos son:

\section{Almidones y sus derivados}

Los gránulos de almidón contienen dos tipos de moléculas poliméricas: 1) Amilosa: formada por unidades de cadena lineal de 1,4- $\alpha$-D-glucopiranosa, y 2 ) amilopectina (Figura 2 ), que es una molécula ramificada formada por unidades de $\alpha$-1,6-glucósido. La primera presenta excelentes propiedades para formar películas fuertes, isotrópicas, inodoras, insípidas y sin color (Tabla I). Cuando se adiciona un plastificante, es posible obtener películas con buenas propiedades mecánicas, especialmente las elaboradas con almidones de alta amilosa, sin embargo, el desarrollo mecánico de éstas se ve afectado por la retrogradación, ya que las dobles hélices de la amilosa y amilopectina se entrecruzan endureciendo la película (Fama, Rojas, Goyanes \& Gerschenson, 2005).

\section{Extractos de algas}

Los alginatos son la principal macromolécula extraída de las algas pardas, éstos son obtenidos de las sales sódicas, cálcicas y potásicas del ácido algínico. Presenta uniones con ácido poliurónico conteniendo bloques de ácido poli- $\beta$-Dmanopiranosilurónico y/o bloques ácido poli- $\alpha$-L-gulosa piranosilurónico (Avendaño- Romero et al., 2013). Las películas elaboradas con estos compuestos resultan quebradizas con poca resistencia al agua, sin embargo, tienen la capacidad de reaccionar de manera irreversible con cationes metálicos polivalentes como los iones de calcio para producir polímeros insolubles en agua (Avendaño- Romero, López-Malo \& Paolu, 2013). El calcio tiene la propiedad de entrecruzarse con el alginato por puentes de hidrógeno, con el ácido poli- $\alpha-\mathrm{L}-$ gulosa piranosil urónico y dependiendo de la concentración usada, puede dar origen a películas con resistencia al agua, 

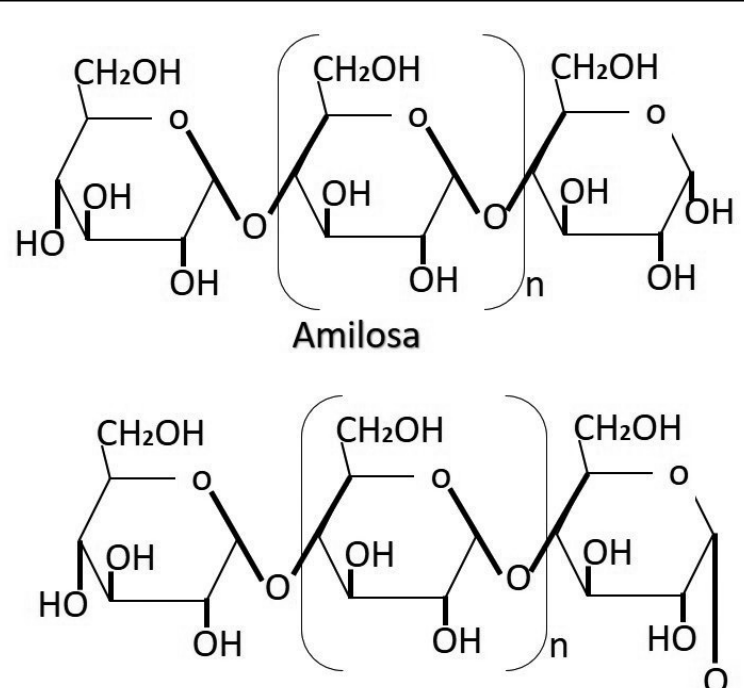

Amilopectina

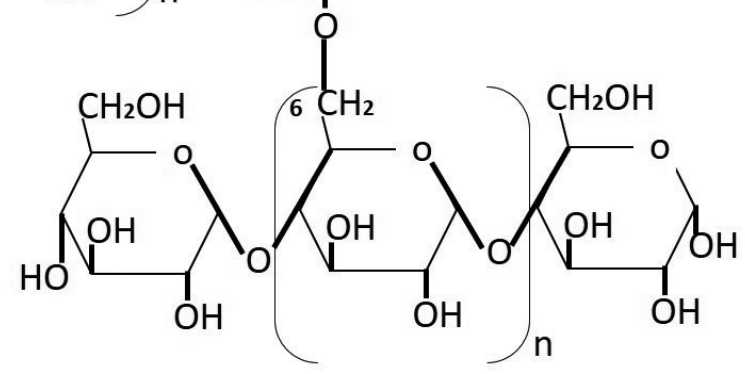

Figura 2. Estructura química de la Amilosa y amilopectina. Fuente: Badui Dergal \& Cejudo Gómez (2006).

\begin{tabular}{|l|l|l|}
\hline \multicolumn{1}{|c|}{ Autor } & \multicolumn{1}{|c|}{ Nombre } & \multicolumn{1}{c|}{ Características } \\
\hline Meza-Velázquez et al., (2013) & $\begin{array}{l}\text { Efecto de una película de hidroxipropil } \\
\text { metilcelulosa-parafina en melón Cantaloup } \\
\text { (Cucumis melo) almacenado en frío }\end{array}$ & $\begin{array}{l}\text { Disminución de la lipasa } \\
\text { Reducción del 50\% de daños por frío } \\
\text { Sin afectaciones por pérdida de peso }\end{array}$ \\
\hline Ortega-Toro et al., (2016) & $\begin{array}{l}\text { Películas de Almidón termoplástico influencia } \\
\text { de la incorporación de hidroxipropil-metil- } \\
\text { celulosa y ácido cítrico }\end{array}$ & $\begin{array}{l}\text { Agregar ácido cítrico disminuyó la } \\
\text { permeabilidad al vapor de agua. La adición } \\
\text { de ácido proporcionó mejor apariencia }\end{array}$ \\
\hline $\begin{array}{l}\text { Echeverri, Montoya,Zuluaga, Castro } \\
\text { \& Gañán, 2011 }\end{array}$ & $\begin{array}{l}\text { Películas de almidón de papa reforzadas con } \\
\text { celulosa bacteriana }\end{array}$ & $\begin{array}{l}\text { Se observó incremento en el módulo de } \\
\text { elasticidad y resistencia a la tracción }\end{array}$ \\
\hline
\end{tabular}

Tabla I. Características obtenidas en películas elaboradas a base de polisacáridos (almidón y celulosa).

sin embargo, la reticulación es tan rápida que es difícil su elaboración (Olivas \&Barbosa-Cánovas, 2008). Las carrageninas, son extraídas de las algas rojas y son una mezcla compleja de diversos polímeros de galactosa, que son solubles en agua. Existen tres principales fracciones, que difieren en el contenido de sulfato de éster y la distribución de residuos de 3,6-anhidro- $\alpha$-D-galactopiranosil. La K-carragenina (Figura 3) es la única con menos cargas negativas por disacárido, presentando excelentes propiedades para formar geles $\mathrm{y}$ películas (Seol, Lim, Jang, Jo \& Lee, 2009).

\section{Otros hidrocoloides}

Entre éstos hidrocoloides se encuentran el tragacanto que es obtenido de Astragalus gummife; la goma arábiga, obtenida de las acacias y la de karaya que se obtiene de árboles de la especie Sterculia urens, originarios de la India. También existen las procedentes de semillas, como las de algarrobo y la guar y finalmente, las obtenidas por fermentación microbiana como la xantana y gelana. Éstas han sido estudiadas en combinación con almidones, alginatos y mezclas con glucósidos (Flores, Costa, Yamashita, Gerschenson \& Grossmann, 2010). La estructura 

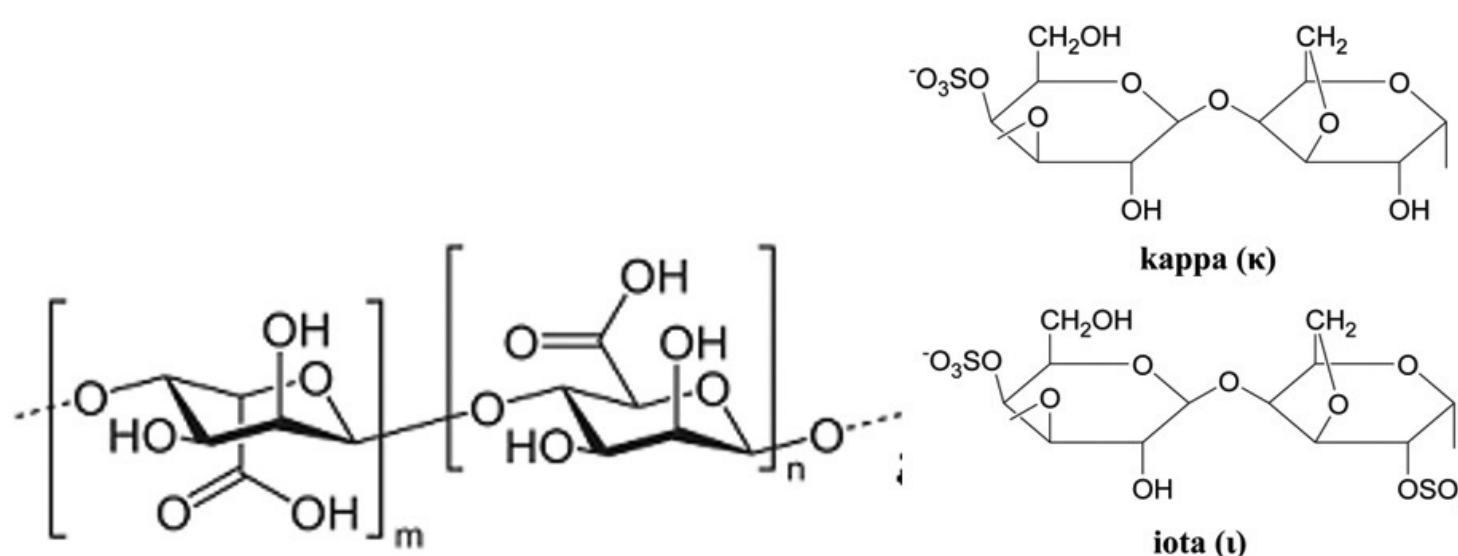

kappa (к)

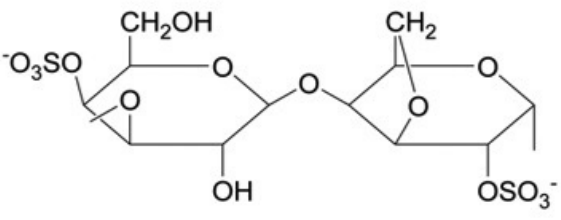

iota (l)

a) Alginato

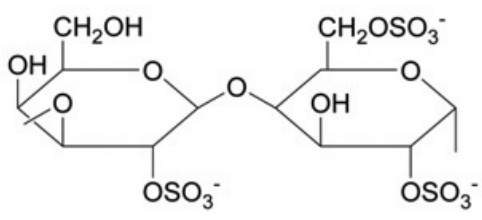

lambda $(\lambda)$

\section{b) Carrageninas}

Figura 3. Estructura química de A) alginato B) formas de carragenina Fuente: McMurry (2004).

química de estos hidrocoloides es compleja, por ejemplo, la goma arábiga se encuentra en la naturaleza como una sal neutra de un polisacárido que contiene $\mathrm{Ca}^{+2}, \mathrm{Mg}^{+2}$ y K $\mathrm{K}^{+2}$. Es una cadena de unidades de $\beta$-galactopiranosa unida en 1,3 con 1,6-galactopiranosa, con residuos de ácido glucorónico o 4-metil glucorónico (Mendoza, Gómez, Hernández, Rodríguez \& Chavarría, 2014). La hidrólisis completa de la molécula produce los cuatro constituyentes básicos: D-galactosa, L-arabinosa, L-ramnosa y D-ácido glucorónico cuyas proporciones son variables y presenta una masa molar que va de 250000 a un millón de Daltons. La forma de la molécula es una espiral corta y rígida (Liu, Qin, He \& Song, 2009).

\section{Pectinas}

Estos polímeros, son producidos ampliamente por las plantas y están compuestos por el ácido 1-4 $\alpha$-D-galactopiranosilurónico, naturalmente esterificados con metanol. De acuerdo con el contenido de metil ésteres o el grado de esterificación, las pectinas se dividen en: de alto (GE $>50 \%$ ) o bajo metoxilo ( $\mathrm{GE}<50 \%$ ), valores decisivos para la solubilidad y propiedades de solidificación (Prasad \& Kochhar 2014).

\section{Quitosano}

Es un polisacárido, que se obtiene por desacetilación de la quitina obtenida a partir de los desechos de los crustáceos, en el exoesqueleto de los insectos y en la pared celular de los hongos (Aider, 2010), está compuesto de dos subunidades, la D-glucosamina y la N-acetil-D-glucosamina, unidas por un enlace glucosídico $ß-(1,4)$. Posee propiedades antimicrobianas y antimicóticas (Aider, 2010) debido principalmente a la presencia de grupos amino cargados positivamente, lo que provoca el deterioro de las proteínas que forman parte de la membrana de los microorganismos, por lo que tiene diversos usos en la agricultura, biomedicina, alimentos, tratamientos de aguas residuales, de potabilización y en la industria de los cosméticos. Esto debido a que posee grupos amino libres, que le confieren mejores propiedades químicas y físicas (López, Rivas, Loaiza \& Sabino, 2010).

Tiene la capacidad de formar películas con excelentes propiedades mecánicas y permeabilidad selectiva a gases, sin embargo, la alta permeabilidad al vapor de agua, limita su aplicación. Debido a la capacidad de formación de películas, es utilizado para mejorar la calidad y extender la vida de anaquel de frutas y hortalizas frescas y procesadas (Loaiza, Chávez \& Sabino, 2014).

\section{Proteínas}

Estos compuestos presentan la habilidad de formar películas y recubrimientos cuyas características dependen de la masa molar, conformación, propiedades eléctricas, flexibilidad y estabilidad térmica (Díaz-González, 2015). Durante la elaboración de éstas, 
es necesario considerar la temperatura y el $\mathrm{pH}$, ya que cualquier modificación podría desnaturalizar la proteína y modificar sus propiedades. Los recubrimientos y películas obtenidos de proteínas, son transparentes y flexibles cuando están elaborados con base de agua y presentan buenas propiedades mecánicas y de barrera, comparadas con las que están hechas a base de polisacáridos y lípidos (Jongjareonrak, Benjakul, Visessanguan \& Tanaka, 2008). Las proteínas al tener una estructura única, presentan un amplio intervalo de propiedades funcionales, especialmente un alto potencial de uniones moleculares; sin embargo, la resistencia al vapor de agua es baja (Huff, 2008). Estas propiedades pueden modificarse por métodos químicos y enzimáticos, realizando mezclas con materiales hidrofóbicos, con algunos polímeros o bien utilizando métodos como la coacervación simple o compleja, la coagulación térmica, la fusión y la solidificación lipídica (Carpiné, Dagostin, Bertan \& Mafra, 2015), o bien mediante el uso de métodos físicos como frotación con aire comprimido, extrusión y cohesión (Montalvo, López-Malo \& Palou, 2012). Las proteínas de la leche así como las provenientes del maíz, trigo y/o soya (Tabla II), son utilizadas por su calidad nutricional y excelentes propiedades sensoriales y funcionales (Campos, Gerschenson \& Flores, 2011; McHugh, 2000).

\section{Películas bioactivas}

Se les denominan recubrimientos o películas bioactivas o inteligentes cuando los recubrimientos o películas son capaces de cambiar las condiciones del alimento, extender la vida de anaquel, mejorar la seguridad microbiológica o las propiedades sensoriales, además de monitorear y ofrecer información acerca de la calidad durante el transporte y el almacenamiento (Puligundla, Jung \& Ko, 2012), éstas también pueden integrar ingredientes con actividad funcional como antioxidantes, vitaminas, prebióticos y probióticos (Falguera, Quintero, Jiménez, Muñoz \& Ibarz, 2011).

\section{Peliculas y Recubrimientos CON PRopiedades ANTIMICROBIANAS}

Dentro de la innovación en la investigación y desarrollo de películas, se encuentran aquellas que son capaces de controlar y liberar agentes antimicrobianos. Esta protección es debida a la neutralización de reacciones o a la interacción de sistemas complejos en los alimentos, los cuales son incorporados para extender la vida de anaquel y prevenir el crecimiento y la diseminación fúngica y bacteriana y de esta manera, lograr minimizar el uso de aditivos (Ortega-Toro, Muñoz, Talens \& Chiralt, 2016). Las películas y recubrimientos comestibles que integran agentes antimicrobianos tienen como objetivo extender la fase lag, suprimiendo el ritmo de crecimiento de los microorganismos (Ávila-Sosa et al., 2012). Los mecanismos por los que son incorporados estos agentes siguen distintas vías, como la incorporación de agentes antimicrobianos volátiles y no volátiles directamente dentro de polímeros o bien, como capas de adsorbentes antimicrobianos dentro de las superficies de polímeros e inmovilizadores antimicrobianos, unidos por enlaces iónicos o covalentes (Ortega-Toro, Muñoz, Talens $\&$ Chiralt, 2016). Por la forma en que se llevan a cabo los mecanismos antimicrobianos, estos se dividen en dos grupos: el primero, de migración de compuestos activos, que pasan parcial o totalmente al alimento o a la superficie y el segundo mediante mecanismos no migratorios, que contienen compuestos con actividad antimicrobiana en la superficie de la película o cubierta (Vázquez-Briones \& Guerrero-Beltràn, 2013). Las sustancias que son utilizadas como agentes antimicrobianos en las películas o recubrimientos, incluyen las sales de sorbato de potasio, ácidos orgánicos como el ácido cinámico (Sharma \& Rao, 2015) aceites esenciales como el de la canela (Ávila-Sosa et al., 2012), extractos de plantas como el de orégano y tomillo (Solano \& de Rojas-Gante, 2012), enzimas como la lisozima (Díaz-González, 2015), bacteriocinas como la nisina y pediocina (Narsaiah et al., 2015) y antibióticos como la natamicina (Fuciños et al., 2015), o bien cationes metálicos, como la plata, que tiene una gran capacidad antimicrobiana tanto en bacterias Gram $(+)$, como en Gram (-), presenta baja volatilidad y toxicidad en células eucariotas, por lo que son utilizadas para el desarrollo de superficies antimicriobianas, en contenedores asépticos y empaques activos, en donde son liberados iones $\mathrm{Ag}^{+}$en bajas

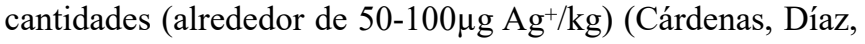
Meléndrez, Cruzat \& García-Cancino, 2009).

\section{Películas y recubrimientos inteligentes}

Las películas y recubrimientos inteligentes son aquellas que permiten hacer un seguimiento de trazabilidad de un producto, esto es desde la cosecha, almacenamiento, venta y consumo (Rodriguez Sauceda et al., 2014). En este tipo de películas se incorpora algún sistema que monitorea y comunica información útil de las propiedades y/o estado del alimento. Éstas se pueden encontrar adheridas al alimento como etiquetas con bio sensores o bien como parte del empaque, recubrimiento o película (Ortega-Toro, Muñoz, Talens \& Chiralt, 2016), y

\begin{tabular}{|l|l|l|l|}
\hline \multicolumn{1}{|c|}{ Materia prima } & \multicolumn{1}{c|}{ Autores } & \multicolumn{1}{c|}{ Proteína } & \multicolumn{1}{c|}{ Propiedades } \\
\hline Zeína & Mendoza \& Caballero (2006) & Vegetal & Brillantes, impermeables \\
\hline Gluten de trigo & Gontard, Duchez, Cuq \& Guilbert (1994) & Vegetal & Baja permeabilidad a los gases \\
\hline Suero de leche & Rossi-Márquez et al. (2009) & Animal & Buena flexibilidad \\
\hline Caseína & Atarés, Bonilla \& Chiralt (2010) & Animal & Transparencia \\
\hline
\end{tabular}

Tabla II. Propiedades que obtienen las películas de distintas fuentes de proteína. 
pueden incorporarse como a) atados a la cadena del polímero, que son películas con partículas funcionales como moléculas poliméricas complejas, en forma de micelas o bien en partículas de microgel, b) ensambles de capa sobre capa; este diseño es utilizado cuando existe la interacción entre las capas, por ejemplo, polímeros o partículas unidas mediante puentes de hidrógeno o interacciones electrostáticas, c) bloques de copolímeros y entrecruzamiento, que se utilizan para películas porosas y d) con diversas combinaciones, preparadas por polímeros y materiales inorgánicos (Tokarev, Motornov \& Minko, 2009).

La morfología y las propiedades de estas películas pueden ser modificados y ajustados según la combinación de la estructura química de los ingredientes y las interacciones entre las interfaces, algunos pueden ser diseñados en estructuras 2D y $3 \mathrm{D}$, como es el caso de las cápsulas de partículas coloidales, de igual forma se pueden integrar sistemas multifuncionales utilizando el enfoque bottom-up (Tokarev, Motornov \& Minko, 2009).

También pueden utilizarse dispositivos que detecten y muestren los cambios en las películas, para esto se recurre a moléculas o sustancias estímulo-sensibles. Estos dispositivos tienen distintas funciones como:

a) Indicadores de tiempo-temperatura, que tienen como objetivo estimar la calidad e integridad del producto, mostrando que el producto ha sido expuesto a temperaturas nivel crítico, por debajo o encima del valor del umbral, esto quiere decir que hay un límite mínimo y máximo para que los indicadores reaccionen y den la información pertinente, estos actúan por medio de reacciones fisicoquímicas o enzimáticas (CortésTapia, 2007).

b) Indicadores de gases en la atmósfera del alimento, que son utilizados para mantener y determinar algún intercambio de gases como el oxígeno, el dióxido de carbono o bien el etileno presente en las atmósferas controladas, para acelerar o retardar la maduración (Cortés-Tapia, 2007).

c) Indicadores de calidad microbiológica; los cuales se basan en la identificación de diferentes metabolitos volátiles generados por el crecimiento microbiano, tales como dióxido de carbono, acetaldehído, amoníaco y ácidos grasos, así como a la variación de acidez $(\mathrm{pH})$ debido al crecimiento microbiano (McMurry, 2004), para determinar estos cambios, son utilizados como parámetros de referencia las variaciones de color, por lo que se agregan pigmentos naturales dentro de la matriz de la película o por fuera de ésta, (Maciel, Yoshida \& Franco, 2012).

\section{LA NANOESTRUCTURACIÓN COMO PARTE DE LA ELABORACIÓN DE LAS PELÍCULAS}

Las películas y recubrimientos al encontrarse como emulsiones o dispersiones pueden ser modificadas estructuralmente para obtener mejores condiciones. La nanoestructuración se enfoca en la caracterización, fabricación y manipulación de estructuras biológicas y no biológicas, por debajo de $100 \mathrm{~nm}$. Las estructuras modificadas en esta escala tienen propiedades funcionales únicas (Weiss, Takhistov \& McClements, 2006). Estos sistemas con características estructuradas a nanoescala, tienen propiedades químicas, físicas y biológicas sustancialmente diferentes a las no modificadas, lo que se manifiesta directamente en el alimento. Esta tecnología ofrece múltiples formas para crear películas y recubrimientos comestibles, un ejemplo de éstas son las nanoláminas, que consisten en dos o más láminas de material con dimensiones nanométricas que son colocadas una sobre otra. El método más eficaz es el basado en el LbL (layer by layer), en el que las superficies de las láminas se encuentran cargadas y armadas por múltiples nanoláminas de diferentes materiales. Esta tecnología permite el control preciso del grosor y propiedades interfaciales de la película, que pueden ser desde 1 hasta $100 \mathrm{~nm}$ por lámina. El nanolaminado ofrece algunas ventajas en la preparación de cubiertas comestibles sobre las técnicas convencionales de elaboración, mejorando la textura y sirviendo como acarreadores de agentes funcionales como colorantes, saborizantes, antioxidantes, nutrientes y agentes antimicrobianos (Weiss, Takhistov \& McClements, 2006).

La composición, el grosor, la estructura y las propiedades del nanomultilaminado de los recubrimientos formados alrededor del alimento, puede ser controlado por diversas vías cambiando el tipo de sustancias adsorbentes en la disolución; esto es, que la adsorción sobre la superficie del alimento dependa tanto de la naturaleza del alimento como de la primera capa colocada sobre éste, realizándose esta interacción por puentes de hidrógeno, fuerzas electrostáticas, interacciones hidrofóbicas o incompatibilidad termodinámica. También se logra, cambiando el orden en el que es sumergido el alimento, la disolución, la dispersión o las condiciones ambientales utilizadas, así como el $\mathrm{pH}$, la fuerza iónica, la constante dieléctrica o la temperatura (Tokarev, Motornov \& Minko, 2009).

Se han elaborado películas que incluyen metales micro y nano estructurados como parte de la matriz, lo que mejora las propiedades de barrera y disminuye la fotodegradación de la película, además presenta actividad antimicrobiana. Estos metales son incorporados a películas y recubrimientos comestibles debido a su capacidad de acarrear oxígeno y oxidar etileno, extendiendo así la vida de anaquel (Llorens, Lloret, Picouet, Trbojevich \& Fernández, 2012). El cobre, el zinc y el titanio nanoestructurados son seguros y pueden ser utilizados en empaques y películas aplicadas a los alimentos (Gruére, 2012); El óxido de zinc nanoestructurado presenta propiedades antimicrobianas; el cobre ha demostrado ser un eficiente sensor de humedad y el óxido de titanio es un bloqueador de UV y presenta resistencia a la abración (Falguera, Quintero, Jiménez, Muñoz \& Ibarz, 2011). La efectividad microbiológica se da por la migración de los cationes a la matriz polimérica (Cárdenas, Díaz, Meléndrez, Cruzat \& García-Cancino, 2009). 
En películas comestibles, la nanotecnología ha sido utilizada con el fin de mejorar sus propiedades bifuncionales. AguirreCárdenas et al., (2011) elaboró y caracterizó películas biodegradables a base de nanobiopartículas de mucílago y pectinas de nopal utilizando una molienda con martillos, lo que permitió la generación de partículas con tamaños menores a $300 \mathrm{~nm}$ y obtuvo las películas más transparentes, resistentes, flexibles, permeables a gases que las elaboradas con películas cuyos materiales no fueron modificados.

También se han realizado películas protectoras y plásticos que incluyen nanocompuestos de polímeros/silicatos, en las que se utilizó la síntesis del compuesto en la etapa de polimerización con el complejo in situ y con el monómero líquido (Carneiroda-Cunha et al., 2010). Los nanocompuestos pueden ser preparados a partir de disoluciones del polímero y el complejo. Éstos ofrecen mayor resistencia al calor, mejores valores del módulo elástico, baja viscosidad, estabilidad dimensional y buena apariencia de superficie(Weiss, Takhistov \& McClements, 2006). Adicionalmente, los nanocompuestos pueden mostrar mejores propiedades de barrera, que se traducen en tasas de transmisión de oxígeno más bajas. A estos se les llaman nanocompuestos de Nylon-6 (Verma, Singh \& Vikas, 2012). La nanotecnología en el campo de los alimentos busca hacer una diferencia importante en el área de cárnicos, por lo que se han integrando péptidos bioactivos, nanoemulsiones de grasa a base de antioxidantes, nanosensores y nanotraceadores de bioseguridad en productos cárnicos con funciones definidas (López-Vázquez et al., 2012). La nanotecnología que elabora estructuras a nanoescala, puede mejorar la interacción de moléculas individuales, evitando reacciones de rancidez y de Maillard (López-Vázquez, Brunner \& Siegrist, 2012).

\section{Aditivos para la elaboración de películas y RECUBRIMIENTOS COMESTIBLES}

En la elaboración de películas y recubrimientos comestibles son incorporados otros componentes que ayudan a mejorar sus propiedades, entre estos se encuentran los plastificantes, los surfactantes, los emulsionantes, los antioxidantes y los reafirmantes de la textura como el glicerol, sorbitol, polietilenglicol, goma gelana, entre otros. Se ha demostrado que algunos aditivos actúan más efectivamente en los alimentos cuando son aplicados formando parte de un recubrimiento o película comestible, que cuando son aplicados en disoluciones acuosas mediante dispersión o inmersión, ya que las coberturas pueden mantener los aditivos en la superficie del alimento durante mayor tiempo (May Gutiérrez, 2015).

Los plastificantes son moléculas de baja masa molary volatilidad y con naturaleza química similar a la del polímero formador del recubrimiento. Éstos son utilizados para mejorar la flexibilidad y la funcionalidad de las películas y recubrimientos. Dentro de los agentes plastificantes más frecuentemente utilizados se encuentran: el glicerol y el sorbitol, que ayudan a mejorar las propiedades mecánicas, así como la permeabilidad al vapor de agua, propiedades térmicas y algunas veces el color. El uso de altas concentraciones de ambos plastificantes incrementa el porcentaje de elongación (Bósquez-Molina \& Vernon-Carter, 2005). Se ha observado que una mayor cantidad de glicerol permite mejor flexibilidad, sin embargo, la permeabilidad al vapor de agua disminuye (Rezaei \& Motamedzadegan, 2015). Generalmente en las formulaciones a base de polisacáridos y de proteínas se requieren de plastificantes como el glicerol para aumentar la flexibilidad de los recubrimientos, también aumenta el volumen libre o la movilidad molecular de los polímeros, reduciendo los enlaces de hidrógeno internos entre las cadenas de polímeros y aumenta el espacio intermolecular. Los plastificantes afectan la capacidad de atracción de agua del sistema y la permeabilidad al oxígeno de los recubrimientos comestibles (Sothornvit \& Krochta, 2000).

El polivinil alcohol (PVA) es un polímero soluble en agua, no tóxico que aporta flexibilidad y permite una buena formación de las películas. Se ha reportado que el uso del PVA en mezcla con el quitosano y la nisina, son efectivos para controlar el crecimiento microbiológico (Wang et al., 2015), este aditivo es comunmente utilizado en películas y empaques funcionales. En la Tabla III se observan los frutos en los que se han utilizado y su funcionalidad sobre éstos.

Otros aditivos incorporados en las películas y recubrimientos comestibles son las sales de calcio, que actúan como agentes

\begin{tabular}{|l|l|l|l|}
\hline Tipo de Fruta & \multicolumn{1}{|c|}{ Matriz de la P/R } & \multicolumn{1}{c|}{ Plastificantes y aditivos } & \multicolumn{1}{c|}{ Función en la P/R } \\
\hline Mango & CMC & Lecitina, PEG, AC & Mantenimiento del color \\
\hline Fresa & $\begin{array}{l}\text { CPS + Caseína + Péctina }+ \\
\text { Alginato }\end{array}$ & $\begin{array}{l}\text { Glicerol, aceite de girasol, AA, } \\
\text { Cloruro de Calcio }\end{array}$ & $\begin{array}{l}\text { Reducción del pardeamiento y prevención } \\
\text { del crecimiento fúngico }\end{array}$ \\
\hline Manzana & $\begin{array}{l}\text { CPS, APS Carragenato, Alginato, } \\
\text { gelana }\end{array}$ & $\begin{array}{l}\text { Glicerol PEG, AA, AO, Aceite } \\
\text { de girasol, CAB, Ncys }\end{array}$ & $\begin{array}{l}\text { Mantenimiento de la textura y color, } \\
\text { reducción de pérdida de humedad, barrera } \\
\text { a los gases, efecto antifúngico }\end{array}$ \\
\hline
\end{tabular}

CMC: Carboximetilcelulosa; AC: Ácido cítrico; AA: Ácido ascórbico; PEG: Polietilenglicol, AO: Ácido oxálico; Ncyst: N-Acetilcisteina; CAB: Cera de abeja; CPS: Concentrado de proteínas de suero lácteo; APS: aislado de proteínas de suero lácteo. Fuente: (May-Gutiérrez, 2015).

Tabla III. Recubrimientos comestibles utilizados en frutas frescas. 
texturizantes, y que aumentan la resistencia mecánica, los agentes antioxidantes que ayudan a prevenir el oscurecimiento en productos susceptibles de pardeamiento (ácido cítrico, ácido ascórbico, cisteína, glutatión) y los saborizantes, colorantes, nutracéuticos y agentes probióticos que pueden mejorar las propiedades sensoriales o nutricionales de trozos de frutas y vegetales enteros o mínimamente procesados (Montero-Calderón, Rojas-Grau, Soliva-Fortuny \& MartínBelloso, 2009).

Los agentes plastificantes como alcoholes polihídricos, ceras y aceites, se utilizan para mejorar la flexibilidad y elongación del material elaborado. La adición de surfactantes y emulsificantes reduce la actividad del agua superficial y la velocidad en la pérdida de humedad de los alimentos recubiertos (BósquezMolina \& Vernon-Carter, 2005). Los agentes de liberación controlada y los lubricantes como las grasas, los aceites, los emulsificantes, el petrolato, el polietilénglicol y el silicón sirven para prevenir que los alimentos recubiertos se vuelvan viscosos o pegajosos (May-Gutiérrez, 2015; Rhim, 2004).

Existe un impacto positivo al utilizar películas comestibles para evitar el daño mecánico o por microorganismos en fruta y hortalizas (Rojas-Graü, Oms-Oliu, Soliva-Fortuny \& MartínBelloso, 2009) mencionan que agregar antioxidantes en la película ayuda a preservar el fruto y evitar la contaminación por el uso de plásticos; sin embargo, muchas de las opciones para recubrimientos aún siguen en investigación o no se encuentran desarrollados a escala industrial.

\section{Conclusiones}

Los materiales más comunes empleados en la elaboración de películas comestibles y recubrimientos son las proteínas, polisacáridos, lípidos y la combinación de éstos. Se han realizado diversos estudios sobre interacciones entre estos polímeros en el diseño y aplicación de películas con propiedades mecánicas y de barrera mejoradas. La investigación en este campo, ha crecido notablemente encontrando películas cuyos materiales han sido modificados para mejorar aún más el funcionamiento de cada una, encontrando algunas capaces de controlar la atmósfera interna del alimento, aquellas que proporcionan brillo o color, lo que mejora su calidad visual, aquellas que son capaces de evitar contaminación y propagación microbiana, tanto fitopatógena como los dañinos para el ser humano, aquellas cuya tecnología de elaboracion permiten cubrir no sólo alguno de los aspectos anteriores, sino dos o más de ellos. Finalmente la tecnología en este rubro ha crecido de tal manera que se ha propuesto el uso de películas inteligentes, las cuales son capaces de detectar algún fallo en la atmósfera del alimento o contaminación microbiana y ser capaces de informar por medio de señales visuales cualquier alteración o contaminación del producto. Esta serie de mejoras, permitirá una disminución en las pérdidas de futas y hortalizas durante el manejo poscosecha así como en el procesamiento, dándole al consumidor la posibilidad de contar con productos de mejor calidad y mayor vida de anaquel, además de no generar residuos contaminantes y/o tóxicos.

\section{AgradeCIMIENTOS}

Agradecemos al Consejo Nacional de Ciencia y Tecnología el apoyo recibido como beca para el estudio de Doctorado en Ciencias de los Alimentos en el Instituto Politécnico Nacional (Registro 260106).

\section{CONFLicto de INTERESES}

Los autores declaramos que no existe ningún conflicto de intereses.

\section{Referencias}

Aguilar, M. M. (2005). Propiedades físicas y mecánicas de películas biodegradables y su empleo en el recubrimiento de frutos de aguacate. En Tesis de Maestría en Tecnología avanzada (pág. 112). Ciudad de México: Instituto Politécnico Nacional. http:// repositoriodigital.ipn.mx/handle/ 123456789/10573

Aguirre-Cárdenas, M., García-Delgado, P., González-González, R., Jofre Garfias, A. L., Legorreta-Siañez, A.V. \& BuenrostroZagal, J. F. (2011). Desarrollo y evaluación de una película comestible obtenida del mucílago del nopal (Opuntia ficus indica) utilizada para reducir la tasa de respiración de nopal verdura. In: VIII Congreso Iberoamericano de Ingeniería de Alimentos. Lima, Perú 23 al 26 de octubre. 1-5. pp. https:// dialnet.unirioja.es/servlet/articulo?codigo $=4106660$

Aider, M. (2010). Chitosan application for active bio-based films production and potential in the food industry. LWT-Food Science and Technology, 43(6), 837-842. https://doi.org/10.1016/j. lwt.2010.01.021

Arredondo-Ochoa, T. (2012). Diseño de empaques comestibles activos a base de almidón modificado para su posible aplicación en alimentos en fresco (Tesis Maestría). Universidad Autónoma de Querétaro. 1-82. http://hdl.handle.net/123456789/929

Arvanitoyannis, I., Kolokuris, I., Nakayama, A. \& Aiba, S. I. (1997). Preparation and study of novel biodegradable blends based on gelatinized starch and 1, 4-trans-polyisoprene (gutta percha) for food packaging or biomedical applications. Carbohydrate Polymers, 34(4), 291-302. https://doi.org/10.1016/S01448617(97)00085-4

Atarés, L., Bonilla, J. \& Chiralt, A. (2010). Characterization of sodium caseinate-based edible films incorporated with cinnamon or ginger essential oils. Journal of Food Engineering, 100(4), 678-687. https://doi.org/10.1016/j.jfoodeng.2010.05.018

Avendaño-Romero, G., López-Malo,A.\& Paolu, E.(2013). Propiedades del alginato y aplicaciones en alimentos. Temas selectos de Ingeniería de Alimentos, 7(1), 87-96. http://web.udlap.mx/ tsia/files/2013/12/TSIA-71-Avendano-Romero-et-al-2013.pdf

Ávila-Sosa, R., Palou, E., Munguía, M. T. J., Nevárez-Moorillón, G. V., Cruz, A. R. N. \& López-Malo, A. (2012). Antifungal activity by vapor contact of essential oils added to amaranth, chitosan, or starch edible films. International Journal of Food Microbiology, 153(1-2), 66-72. DOI: 10.1016/j. ijfoodmicro.2011.10.017

Badui Dergal, S. \& Cejudo Gómez, H. R. T. (2006). Química de los alimentos. Pearson educación, México 
Bósquez-Molina, E. \& Vernon-Carter, E. J. (2005). Efecto de plastificantes y calcio en la permeabilidad al vapor de agua de películas a base de goma de mezquite y cera de candelilla. Revista Mexicana de Ingeniería Química, 4(2), 157-162. http://www.redalyc.org/articulo.oa?id=62040203

Cagri, A., Ustunol, Z. \& Ryser, E. T. (2004). Antimicrobial edible films and coatings. Journal of Food Protection, 67(4), 833-848. DOI: $10.4315 / 0362-028 X-67.4 .833$

Campos, C.A., Gerschenson, L. N. \& Flores, S. K. (2011). Development of edible films and coatings with antimicrobial activity. Food and Bioprocess Technology, 4(6), 849-875. https://doi. org/10.1007/s11947-010-0434-1

Cárdenas, G., Díaz V.J., Meléndrez, M. F., Cruzat C.C. \& García Cancino, A. (2009). Colloidal $\mathrm{Cu}$ nanoparticles/chitosan composite film obtained by microwave heating for food package applications. Polymer bulletin, 62(4), 511-524. https://doi. org/10.1007/s00289-008-0031-x

Carmona Gallego, J.A., Cordobés, F., Guerrero Conejo, A. F., Martínez, I. \& Partal López, P. (2007). Influencia del pH y de la fuerza iónica sobre la gelificación térmica de proteínas de la yema de huevo. Grasas y aceites, 58(3), 289-296. http://hdl.handle. net/11441/54857

Carneiro-da-Cunha, M. G., Cerqueira, M.A., Souza, B. W., Carvalho, S., Quintas, M.A., Teixeira, J.A. \& Vicente,A.A. (2010). Physical and thermal properties of a chitosan/alginate nanolayered PET film. Carbohydrate Polymers, 82(1), 153-159. https://doi. org/10.1016/j.carbpol.2010.04.043

Carpiné, D., Dagostin, J. L. A., Bertan, L. C. \& Mafra, M. R. (2015). Development and characterization of soy protein isolate emulsion-based edible films with added coconut oil for olive oil packaging: Barrier, mechanical, and thermal properties. Food and bioprocess technology, 8(8), 1811-1823. https://doi. org/10.1007/s11947-015-1538-4

Cortés Tapia, C. P. (2007) Envases inteligentes. Universidad Andrés Bello, repositorio.unab.cl/xmlui/handle/ria/4352.

Díaz-González R. (2015). Films biodegradables antimicrobianos a base de almidón y gelatina. Universitat Politécnica de Valencia, 1-21. hdl.handle.net/10251/56543

Echeverri, N, Montoya, Ú., Zuluaga, R., Castro, C. \& Gañán, P. (2011). Películas de almidón de papa reforzadas con celulosa bacteriana. Revista ReCiTeIA, 11(1) 83-91.

Escobar, D., Sala, A., Silvera, C., Harispe, R. \& Márquez, R. (2009). Películas biodegradables y comestibles desarrolladas en base a aislado de proteínas de suero lácteo: estudio de dos métodos de elaboración y del uso de sorbato de potasio como conservador. Revista del Laboratorio tecnológico del Uruguay, 4, 33-36.

Espino-Díaz, M., De Jesús Ornelas-Paz, J., Martínez-Téllez, M. A., Santillán, C., Barbosa-Cánovas, G. V., Zamudio-Flores, P. B. \& Olivas, G. I. (2010). Development and characterization of edible films based on mucilage of Opuntia ficus-Indica (1.). Journal of Food Science, 75(6). E347-E352. DOI: 10.1111/j.17503841.2010.01661.x

Falguera, V., Quintero, J.P., Jiménez, A., Muñoz, J.A. \& Ibarz,A. (2011). Edible films and coatings: structures, active functions and trends in their use. Trends in Food Science \& Technology, 22(6), 292-303. https://doi.org/10.1016/j.tifs.2011.02.004

Fama, L., Rojas, A. M., Goyanes, S. \& Gerschenson, L. (2005). Mechanical properties of tapioca-starch edible films containing sorbates. LWT-Food Science and Technology, 38(6), 631-639. https://doi.org/10.1016/j.lwt.2004.07.024

FAO. (2018). Despilfarro de alimentos: datos y cifras clave. Food wastage footprint, 1-5. http://www.fao.org/news/story/es/ item/196450/icode/

Flores, S. K., Costa, D., Yamashita, F., Gerschenson, L. N. \& Grossmann, M. V. (2010). Mixture design for evaluation of potassium sorbate and xanthan gum effect on properties of tapioca starch films obtained by extrusion. Materials Science and Engineering: C,30(1), 196-202.https://doi.org/10.1016/j. msec.2009.10.001

Fuciños, C., Míguez, M., Cerqueira, M.A., Costa, M. J., Vicente,A.A., Rúa, M.L.\&Pastrana,L.M.(2015). Functional characterization and antimicrobial efficiency assessment of smart nanohydrogels containing natamycin incorporated into polysaccharide-based films. Food and Bioprocess Technology, 8(7), 1430-1441. https://doi.org/10.1007/s11947-015-1506-z

Gontard, N., Duchez, C., Cuq, J. L. \& Guilbert, S. (1994). Edible composite films of wheat gluten and lipids: water vapour permeability and other physical properties. International Journal of Food Science \& Technology, 29(1), 39-50. https:// doi.org/10.1111/j.1365-2621.1994.tb02045.x

Gruère, G. P. (2012). Implications of nanotechnology growth in food and agriculture in OECD countries. Food Policy, 37(2), 191198. https://doi.org/10.1016/j.foodpol.2012.01.001

Hibberler R.C.(2006). Mecánica de materiales. 6ta. Edición. Pearson Educación. Mexico. ISBN 970-26-0639-3 pag 93-95.https:// archive.org/details/MecnicaDeMateriales Hibbeler8aEdiciin

Hong, S. I., Park, J. D. \& Kim, D. M. (2000). Antimicrobial and physical properties of food packaging films incorporated with some natural compounds. Food Science and Biotechnology, 9(1), $38-42$.

Huff, K. (2008). Active and intelligent packaging: innovations for the future. Department of Food Science \& Technology. Virginia Polytechnic Institute and State University, Blacksburg, Va, 1-13.https://www.iopp.org/files/public/VirginiaTechKarleigh Huff.pdf

Jaworek, A. T. S. A. \& Sobczyk, A. T. (2008). Electrospraying route to nanotechnology: an overview. Journal of electrostatics, 66(34), 197-219. https://doi.org/10.1016/j.elstat.2007.10.001

Jongjareonrak, A., Benjakul, S., Visessanguan, W. \& Tanaka, M. (2008). Antioxidative activity and properties of fish skin gelatin films incorporated with BHT and $\alpha$-tocopherol. Food Hydrocolloids, 22(3), 449-458. https://doi.org/10.1016/j. foodhyd.2007.01.002

Kean, T. \& Thanou, M. (2010). Biodegradation, biodistribution and toxicity of chitosan. Advanced drug delivery reviews, 62(1), 3-11. https://doi.org/10.1016/j.addr.2009.09.004

Liu, F., Qin, B., He, L. \& Song, R. (2009). Novel starch/chitosan blending membrane:Antibacterial, permeable and mechanical properties. Carbohydrate Polymers, 78(1), 146-150. https:// doi.org/10.1016/j.carbpol.2009.03.021

Llorens, A., Lloret, E., Picouet, P. A., Trbojevich, R. \& Fernandez, A. (2012). Metallic-based micro and nanocomposites in food contact materials and active food packaging. Trends in Food Science \& Technology, 24(1), 19-29. https://doi.org/10.1016/j. tifs.2011.10.001

Loaiza, M., Chávez G. \& Sabino M. (2014). Obtención y modificación química de oligosacáridos de quitosano. Revista 
latinoamericana de Metalurgia y Materiales, S6, 25-26.

López, Á., Rivas, J., Loaiza, M. \& Sabino, M. (2010). Degradación de películas plastificadas de quitosano obtenidas a partir de conchas de camarón (L. vannamei). Revista de la Facultad de Ingeniería Universidad Central de Venezuela, 25(2), 133-143. http://www.scielo.org.ve/scielo.php?script=sci_arttext\&pid $=\mathrm{S} 0798-40652010000200014$

López-Vázquez, E., Brunner, T. A. \& Siegrist, M. (2012). Perceived risks and benefits of nanotechnology applied to the food and packaging sector in México. British Food Journal, 114(2), 197-205. https://doi.org/10.1108/00070701211202386

Maciel, V. B., Yoshida, C. M. \& Franco, T. T. (2012). Development of a prototype of a colourimetric temperature indicator for monitoring food quality. Journal of food engineering, 111(1), 21-27. https://doi.org/10.1016/j.jfoodeng.2012.01.037

Maki-Díaz, G., Peña-Valdivia, C. B., García-Nava, R., ArévaloGalarza, M. L., Calderón-Zavala, G. \& Anaya-Rosales, S. (2015). Características físicas y químicas de nopal verdura (Opuntia ficus-indica) para exportación y consumo nacional. Agrociencia, 49(1), 31-51. http://www.scielo.org. $\mathrm{mx} /$ pdf/agro/v49n1/ v49n1a3.pdf

Márquez, C. J., Trillos, O., Cartagena, J. R. \& Cotes, J. M. (2009). Evaluación fisicoquímica y sensorial de frutos de uchuva (Physalis peruviana L.). Vitae, 16(1), 42-48. http://www.scielo. org.co/pdf/vitae/v16n1/v16n1a05.pdf

May Gutiérrez, M. E. (2015). Desarrollo de un recubrimiento comestible a base de mucílago de nopal (Opuntia spp.)(Doctoral dissertation). Universidad Autónoma de Querétaro. ri.uaq.mx/ handle/123456789/2740

McHugh, T. H. (2000). Protein-Lipid interactions in edible films and coatings. Nahrung, 44: 148-151. DOI: 10.1002/1521-3803 (20000501)44:3<148::AID-FOOD148>3.0.CO;2-P

McMurry, J (2004). Química Orgánica. Ed Cengage learning, México D. F.

Mendoza, M. \& Caballero, J. I. M. (2006). Estudio y desarrollo de films a base de zeína de maíz, con distintos tipos de plastificantes. Alimentaria: Revista de tecnología e higiene de los alimentos, (372), 86-87.

Mendoza, B., Gómez, E., Hernández, E., Rodríguez, A. \& Chavarría, N. (2014). Elaboración y caracterización de películas biodegradables a partir de mucílago de nopal-caseinato de sodio y mucílago de nopal-pectina. In Ciencias Agropecuarias Handbook T-II: Congreso Interdisciplinario de Cuerpos Académicos (pp. 129-136). ECORFAN.

Meza Velázquez, J. A., Guzmán, G. A., García Díaz, C. L., Fortis, H. M., Preciado Rangel, P. \& Esparza Rivera, J. R. (2013). Effect of a film of hidroxypropyl methylcellulose- paraffin in Cantaloupe melon (Cucumis melo) stored in cold. Revista Mexicana de Ciencias Agricolas, 4(2),259-271. http://www. scielo.org.mx/pdf/remexca/v4n2/v4n2a6.pdf

Miranda, S. P., Cárdenas, G., López, D. \& Lara-Sagahon, A. V. (2003). Comportamiento de películas de Quitosán compuesto en un modelo de almacenamiento de aguacate. Revista de la Sociedad Química de México, 47(4), 331-336. http://www.scielo.org. $\mathrm{mx} / \mathrm{pdf} / \mathrm{rsqm} / \mathrm{v} 47 \mathrm{n} 4 / \mathrm{v} 47 \mathrm{n} 4 \mathrm{a} 8 . \mathrm{pdf}$

Monroy-Villagrana, A., Cano-Sarmiento, C., Alamilla-Beltrán, L., Hernández-Sánchez, H. \& Gutiérrez-López, G. F. (2014). Coupled taguchi-rsm optimization of the conditions to emulsify $\alpha$-tocopherol in an Arabic gum-maltodextrin matrix by microfluidization. Revista Mexicana de Ingeniería Química, 13(3), 679-688 http://www.scielo.org.mx/pdf/rmiq/ v13n3/v13n3a3.pdf

Montalvo, C., López-Malo,A.\& Palou, E. (2012). Películas comestibles de proteína: características, propiedades y aplicaciones. Temas selectos de ingeniería de alimentos, 6(2), 32-46. http://web. udlap.mx/tsia/files/2013/12/TSIA-62Montalvo-et-al-2012.pdf

Montero-Calderón, M., Rojas-Graü, M. A., Soliva-Fortuny R. \& Martín-Belloso, O. (2009). Tendencias en el procesado mínimo de frutas y hortalizas frescas. Internal quality profile and influence of packaging conditions on fresh-cut pineapple, 69(3), 48-51.http://www.horticom.com/revistasonline/extras/ extra09/48 51.pdf

Narsaiah, K., Wilson, R. A., Gokul, K., Mandge, H. M., Jha, S. N., Bhadwal, S., Anurag R.K. Malik R.K. \& Vij, S. (2015). Effect of bacteriocin-incorporated alginate coating on shelf-life of minimally processed papaya (Carica papaya L.). Postharvest Biology and Technology, 100,212-218. https:// doi.org/10.1016/j.postharvbio.2014.10.003

Olivas, G. I. \& Barbosa-Cánovas, G. V. (2008). Alginate-calcium films: water vapor permeability and mechanical properties as affected by plasticizer and relative humidity. LWT-Food Science and Technology, 41(2), 359-366. https://doi.org/10.1016/j. lwt.2007.02.015

Ortega-Toro, R., Muñoz, A., Talens, P. \& Chiralt, A. (2016). Improvement of properties of glycerol plasticized starch films by blending with a low ratio of polycaprolactone and/ or polyethylene glycol. Food Hydrocolloids, 56, 9-19. https:// doi.org/10.1016/j.foodhyd.2015.11.029

Prasad, P. \& Kochhar, A. (2014). Active packaging in food industry: a review. Journal of Environmental Science, Toxicology and Food Technology, 8(5), 1-7. DOI: 10.9790/2402-08530107

Puligundla, P., Jung, J. \& Ko, S. (2012). Carbon dioxide sensors for intelligent food packaging applications. Food Control, 25(1), 328-333. https://doi.org/10.1016/j.foodcont.2011.10.043

Ramos-García, M. D. L., Bautista-Baños, S., Barrera-Necha, L. L., Bósquez-Molina, E., Alia-Tejacal, I. \& Estrada-Carrillo, M. (2010). Compuestos antimicrobianos adicionados en recubrimientos comestibles para uso en productos hortofrutícolas. Revista mexicana de fitopatología, 28(1), 44-57.http://www.scielo.org.mx/pdf/rmfi/v28n1/v28n1a5.pdf

Rezaei, M. \& Motamedzadegan,A. (2015). The Effect of Plasticizers on Mechanical Properties and Water Vapor Permeability of GelatinBased Edible Films Containing Clay Nanoparticles. World Journal of Nano Science and Engineering, 5(04), 178. DOI: 10.4236/wjnse.2015.54019

Rhim, J. W. (2004). Physical and mechanical properties of water resistant sodium alginate films. LWT-Food science and technology, 37(3), 323-330. https://doi.org/10.1016/j. lwt.2003.09.008

Rodríguez-Sauceda, R., Rojo-Martínez, G. E., Martínez-Ruiz, R., Piña-Ruiz, H. H., Ramírez-Valverde, B., Vaquera-Huerta, H. \& Cong-Hermida, M. D. L. C. (2014). Envases inteligentes para la conservación de alimentos. Ra Ximhai, 10(6), 151-173. http://www.redalyc.org/pdf/461/46132135012.pdf

Rojas-Graü, M., Oms-Oliu, G., Soliva-Fortuny, R. \& Martín-Belloso, O. (2009). The use of packaging techniques to maintain freshness in 
fresh-cut fruits and vegetables: a review. International Journal of Food Science \& Technology, 44(5), 875-889. https://doi. org/10.1111/j.1365-2621.2009.01911.x

Rossi-Márquez, G., Han, J.H., García-Almendárez, B., Castaño-Tostado, E. \& Regalado-González, C. (2009). Effect of temperature, pH and film thickness on nisin release from antimicrobial whey protein isolate edible films. Journal of the Science of Food and Agriculture, 89(14),2492-2497.https://doi.org/10.1002/jsfa.3751

Ruiz Avilés, G. (2006). Obtención y caracterización de un polímero biodegradable a partir del almidón de yuca. Ingeniería y ciencia, 2(4). 5-28. http://www.redalyc.org/pdf/835/83520401.pdf

Salinas Salazar, V. M., Trejo Márquez, M. A. \& Lira Vargas, A. (2015). Propiedades físicas, mecánicas y de barrera de películas comestibles a base de mucílago de Nopal como alternativa para la aplicación en frutos. Revista Iberoamericana de Tecnología Postcosecha, 16(2), 193-198. http://www.redalyc. org/pdf/813/8134317 6007.pdf

Seol, K. H., Lim, D. G., Jang, A., Jo, C. \& Lee, M. (2009). Antimicrobial effect of $\kappa$-carrageenan-based edible film containing ovotransferrin in fresh chicken breast stored at $5 \mathrm{C}$. Meat Science, 83(3), 479-483. DOI: 10.1016/j.meatsci.2009.06.029

Sharma, S. \& Rao, T. R. (2015). Xanthan gum based edible coating enriched with cinnamic acid prevents browning and extends the shelf-life of fresh-cut pears. LWT-Food Science and Technology, 62(1), 791-800. https://doi.org/10.1016/j. lwt.2014.11.050

Silva-Weiss, A., Ihl, M., Sobral, P. J. A., Gómez-Guillén, M. C. \& Bifani, V. (2013). Natural additives in bioactive edible films and coatings: functionality and applications in foods. Food Engineering Reviews, 5(4), 200-216. https://doi.org/10.1007/ s12393-013-9072-5

Solano, A. C. V. \& de Rojas Gante, C. (2012). Two different processes to obtain antimicrobial packaging containing natural oils. Food and Bioprocess Technology, 5(6), 2522-2528. https://doi. org/10.1007/s11947-011-0626-3

Sothornvit, R. \& Krochta, J. M. (2000). Plasticizer effect on oxygen permeability of $\beta$-lactoglobulin films. Journal of Agricultural and Food Chemistry, 48(12), 6298-6302. DOI: 10.1021/ jf0008361

Tokarev, I., Motornov, M. \& Minko, S. (2009). Molecular-engineered stimuli-responsive thin polymer film: a platform for the development of integrated multifunctional intelligent materials. Journal of Materials Chemistry, 19(38), 6932-6948. DOI:10.1039/B906765E

Trejo, V., Aragón, N. \& Miranda, P. (2001). Estimación de la permeabilidad al vapor de agua en películas a base de quitosán. Revista de la Sociedad Química de México, 45(1), 01-05.http:/www.scielo.org.mx/pdf/rsqm/v45n1/v45n1a1.pdf

Vartiainen, J., Vähä-Nissi, M. \& Harlin, A. (2014). Biopolymer films and coatings in packaging applications - a review of recent developments. Materials Sciences and applications, 5(10), 708. DOI: $10.4236 / \mathrm{msa} .2014 .510072$

Vázquez-Briones, M. C. \& Guerrero-Beltrán, J. A., (2013). Recubrimientos de frutas con biopelículas. Temas Selectos de Ingeniería de Alimentos, 7(2), 5-14. http://web.udlap.mx/ tsia/files/2014/12/TSIA-72-Vazquez-Briones-et-al-2013.pdf

Verma, A. K., Singh, V. P. \& Vikas, P. (2012). Application of nanotechnology as a tool in animal products processing and marketing: an overview. American Journal of Food Technology, 7(8), 445-451. DOI: 10.3923/ajft.2012.445.451

Wang, H., Zhang, R., Zhang, H., Jiang, S., Liu, H., Sun, M. \& Jiang, S. (2015). Kinetics and functional effectiveness of nisin loaded antimicrobial packaging film based on chitosan/poly (vinyl alcohol). Carbohydrate polymers, 127, 64-71. DOI: 10.1016/j. carbpol.2015.03.058

Weiss, J., Takhistov, P. \& McClements, D. J.(2006). Functional materials in food nanotechnology. Journal of Food Science, 71(9),107116. https://doi.org/10.1111/j.1750-3841.2006.00195.x

Yang, L. \& Paulson, A. T. (2000a). Effects of lipids on mechanical and moisture barrier properties of edible gellan film. Food research international,33(7),571-578. https://doi.org/10.1016/ S0963-9969(00)00093-4

Yang, L. \& Paulson, A. T. (2000b). Mechanical and water vapour barrier properties of edible gellan films. Food Research International,33(7), 563-570. https://doi.org/10.1016/S09639969(00)00092-2 